\title{
Different Clinical Presentation and Tissue Characterization in a Monozygotic Twin Pair with MYH7 Mutation-Related Hypertrophic Cardiomyopathy
}

\author{
Jie Wang, ${ }^{1, *}$ BMSci, Weihao Li, ${ }^{1, *}$ BMSci, Yuchi Han, ${ }^{2}$ MD and Yucheng Chen, ${ }^{1}$ MD
}

\begin{abstract}
Summary
This case report demonstrates a pair of monozygotic twins with hypertrophic cardiomyopathy (HCM) carrying the same pathogenic mutation of MYH7 (p.G768R; c.2302G>A), detected by whole exome and Sanger genetic sequencing methods. On multi-modality imaging, they were reported to have similar, but not identical, morphologic expression. Particularly, the clinical presentation and tissue characteristics were not the same. Late gadolinium enhancement (LGE) and T1 mapping of cardiac magnetic resonance showed different extents of myocardial fibrotic characteristics in the twins (twin A: 16.3\% LGE and 32.6\% extracellular volume [ECV] of the whole left ventricle; twin B: 5.4\% LGE and $28.1 \% \mathrm{ECV}$ of the whole left ventricle). This extraordinary case of HCM provides evidence on the complex pathophysiological mechanisms of HCM and suggests the likely impact of epigenetics and environmental factors on HCM phenotype.
\end{abstract}

(Int Heart J 2019; 60: 477-481)

Key words: Late gadolinium enhancement, T1 mapping, Cardiac magnetic resonance, Phenotype

$\mathrm{H}$ ypertrophic cardiomyopathy ( $\mathrm{HCM})$ is an autosomal dominant genetic disease with a prevalence of 1:500 in the general adult population. ${ }^{1)}$ Approximately $60 \%$ of $\mathrm{HCM}$ is caused by the genetic variants encoding myocardial sarcomere proteins, in which more than 1000 distinct mutations in 11 genes have been identified. ${ }^{2,3)}$ Of those, $\beta$-myosin heavy chain $(\mathrm{MYH7})$ is the most commonly affected gene. HCM in monozygotic twins is a rare occurrence. It is interesting to observe HCM in monozygotic twins who share identical genes as it provides a unique opportunity to observe phenotypic differences with the control of genetic heterogeneity and phenotypic diversity can be considered as environmental in origin. HCM is characterized by myocardial fiber disarray and increased extracellular matrix with accumulation of interstitial fibrosis. ${ }^{4)}$ Late gadolinium enhancement imaging (LGE) allows for the identification of focal fibrotic areas that are associated with disease progression and portend a worse prognosis. ${ }^{5,6)}$ Extracellular volume fraction (ECV) helps detect diffuse fibrosis in $\mathrm{HCM} .{ }^{7.8)} \mathrm{Al}-$ though there are a few reports on the association of genotype and phenotype in patients with $\mathrm{HCM},{ }^{9-12)}$ there are no reports to date on the quantitative assessment of myocardial fibrosis using LGE or ECV in monozygotic twins with HCM. Therefore, in this study, we explored the association of genotype and phenotype in monozygotic twins with HCM.

\section{Case Report}

A 49-year-old female patient (twin B, II3) was admitted to the hospital for syncope. Her monozygotic twin sister (twin A, II2) experiencing symptoms of palpitation and exertional dyspnea had been diagnosed as having HCM and atrial fibrillation (AF) 1 year ago. Blood tests revealed elevated cardiac troponin $\mathrm{T}$ (cTnT) level of 19 $\mathrm{ng} / \mathrm{L}$ (reference range, 0-14 ng/L) and an obviously elevated N-terminal pro-brain natriuretic peptide (NT-proBNP) level of $10,214 \mathrm{pg} / \mathrm{mL}$ (reference range, $0-88 \mathrm{pg} /$ $\mathrm{mL})$ in twin A and slightly elevated cTnT (18.1 ng/L) and lower level of NT-pro-BNP $(1516 \mathrm{pg} / \mathrm{mL})$ in twin B. The twins received a comprehensive evaluation, including electrocardiogram (ECG) and multi-modality imaging, at the same time. ECG revealed AF and left bundle branch block (BBB) in twin $\mathrm{A}$ and first-degree atrioventricular block, left anterior hemiblock and right BBB in twin B (Figure 1 A). In addition, twin $\mathrm{B}$ was diagnosed as having sick sinus syndrome (SSS) because of frequent sinus arrest, and her maximal RR interval was 3.3 seconds based on 24hours ambulatory ECG (Figure 2). Echocardiography demonstrated that the twins had both focal ventricular septum hypertrophy and significant bi-atrial enlargement

From the 'Division of Cardiology, West China Hospital, Sichuan University, Chengdu, China and ${ }^{2}$ Cardiovascular Division, Department of Medicine, University of Pennsylvania, Philadelphia, USA.

*These authors contributed equally to this work.

This work was supported by the National Natural Science Foundation of China [grant number 81571638 and 81271531 ].

Address for correspondence: Yucheng Chen, MD, Division of Cardiology, West China Hospital, Sichuan University, Guoxue Xiang No. 37, Chengdu, Sichuan Province 610041, China. E-mail: chenyucheng2003@126.com

Received for publication March 23, 2018. Revised and accepted June 14, 2018.

Released in advance online on J-STAGE February 8, 2019.

doi: 10.1536/ihj.18-167

All rights reserved by the International Heart Journal Association. 
Twin A

Twin B

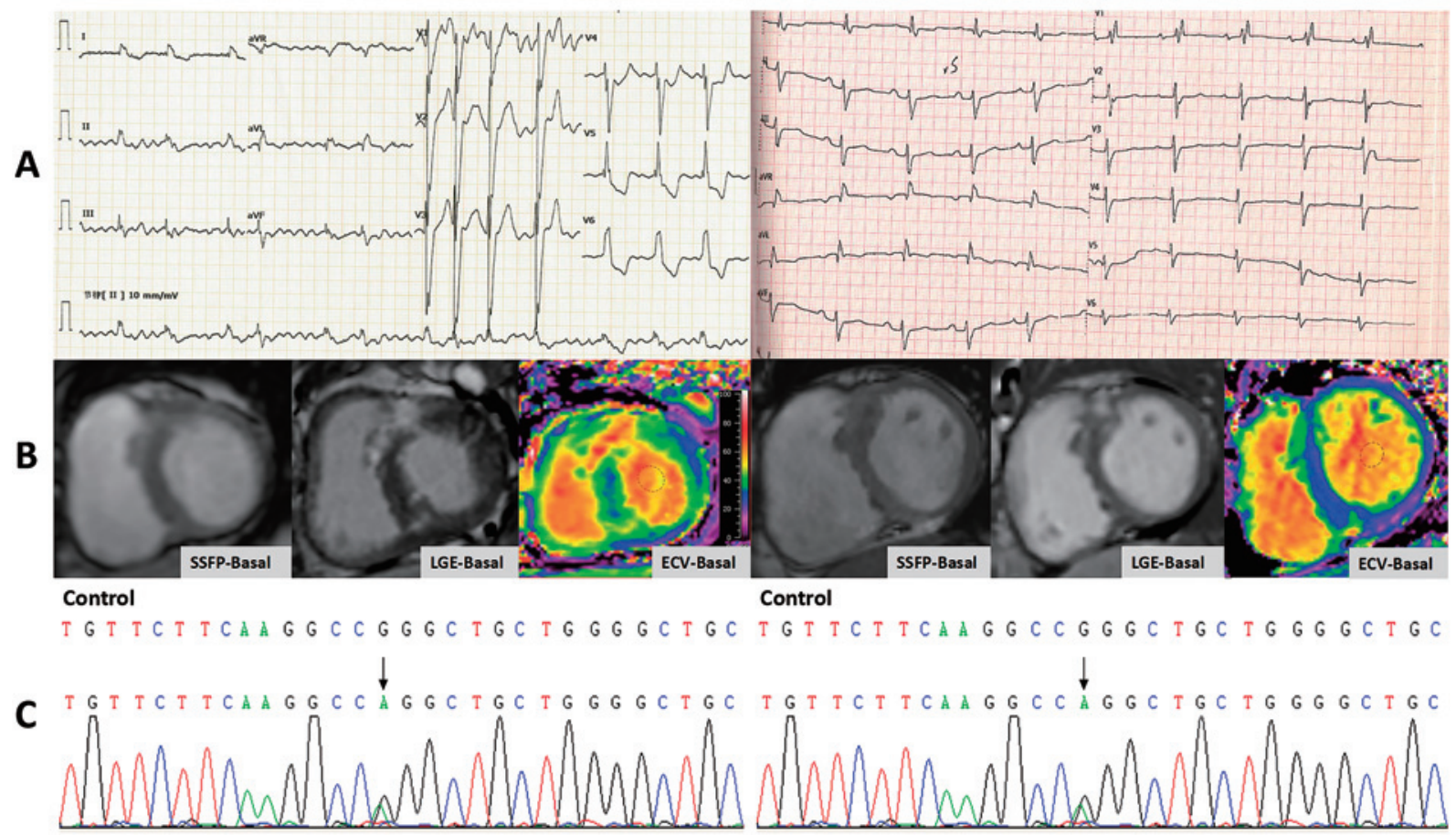

Figure 1. Comparison of clinical and multi-modality imaging characteristics in a monozygotic twin pair with hypertrophic cardiomyopathy. (A: electrocardiogram; B: myocardial fibrotic characteristics quantified by late gadolinium enhancement and extracellular volume; C: single-site mutation of $M Y H 7$ [p.G768R, c.2302G > A] gene)

(twin A: left atrium, $48 \mathrm{~mm}$; right atrium, $43 \mathrm{~mm}$; twin $\mathrm{B}$ : 47 and $42 \mathrm{~mm}$, respectively). Tissue Doppler examination also showed filling abnormality of the left ventricle (LV) with E/e' (lateral) of 30.4 in twin A and 19.7 in twin B. Cardiac magnetic resonance (CMR) confirmed focal LV hypertrophy mainly located in basal anteroseptum with a maximal thickness of $17 \mathrm{~mm}$ in twin $\mathrm{A}$ and $16 \mathrm{~mm}$ in twin $\mathrm{B}$. In addition, the 3 -chambered view revealed no obvious differences in the anterior mitral valve leaflet length (twin A: $29.7 \mathrm{~mm}$; twin B: $30.1 \mathrm{~mm}$ ) and posterior mitral valve leaflet length (twin A: $16.8 \mathrm{~mm}$; twin B: $18.3 \mathrm{~mm}$ ). Therefore, the twins were identified to have similar, but not identical, morphologic expression. Interestingly, there were different degrees of fibrotic burden in the monozygotic twins, when quantified either as LGE proportion (LGE defined as 6 standard deviation from normal) or T1 mapping of CMR (twin A: $16.3 \%$ LGE and $32.6 \%$ extracellular volume [ECV] of the whole LV; twin B: $5.4 \%$ and $28.1 \%$, respectively; Figure $1 \mathrm{~B})$. In addition, the twins were living far away from each other and therefore, had different living environments and life habits, including diet and exercise.

We conducted whole exome sequencing in the twins. Exon-enriched DNA was sequenced by the Illumina hiseq 2500 platform following the manufacturer's instructions. Raw image files were processed using BclToFastq (Illumina) for base calling and generating raw data. The lowquality variations were filtered out using a quality score of $\geq 20$ (Q20). The sequencing reads were aligned to the NCBI human reference genome (hg19) using BWA.
VarScan and GATK were used to analyze single nucleotide polymorphisms (SNPs) and indel of the sequence. Data analysis was applied as follows: (1) Synonymous changes and SNPs with minor allele frequencies higher than 5\% were removed. (2) Nonsynonymous changes were filtered using SIFT software. (3) The function of mutated genes and their association with the disease were analyzed. Genetic results were then confirmed using the Sanger sequencing method. Single site mutation of $M Y H 7$ (p.G768R, c.2302G>A) in twins had been previously reported in $\mathrm{HCM}^{13)}$ (Figure 1C) and was classified as likely pathogenic based according to the guideline ${ }^{14)}$ recommendation. In addition, co-segregation with affected family members was demonstrated for at least one patient. Therefore, this genetic variant was considered as a likely pathogenic mutation related to HCM in our study twins. In addition, clinical screening and genetic testing were offered to other relatives (III, III2, III3, III4, III5, IV1, IV2, IV3, and IV4) of the twins. The pedigree of the twins and other family members is shown in Figure 3. The subjects (II1, III2, III4, and IV1) were also diagnosed as having HCM and identified to be carrying the same p.G768R (c.2302G $>$ A) mutation in the $M Y H 7$ gene. Clinical evaluation and genetic analyses of other family members were normal and considered as being unaffected. Furthermore, cardiac failure in twin A was successfully controlled using beta-adrenergic receptor blockers and diuretics following the diagnosis of HCM. In addition, long-term oral anticoagulation was prescribed for AF. Twin B was recommended a cardioverter-defibrillator (ICD) implantation for 

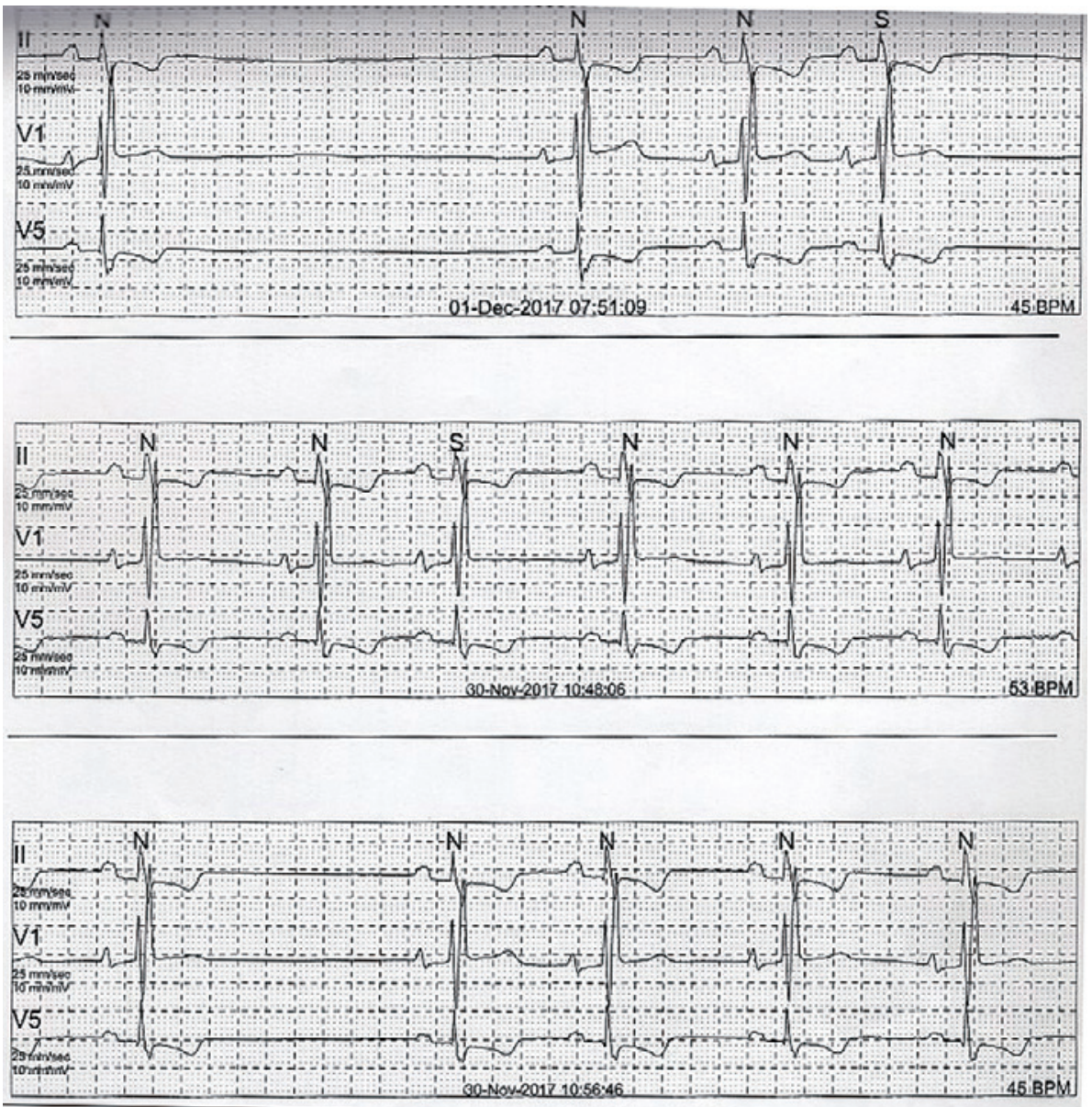

Figure 2. Twenty-four-hour ambulatory electrocardiographic characteristics of twin B.

I

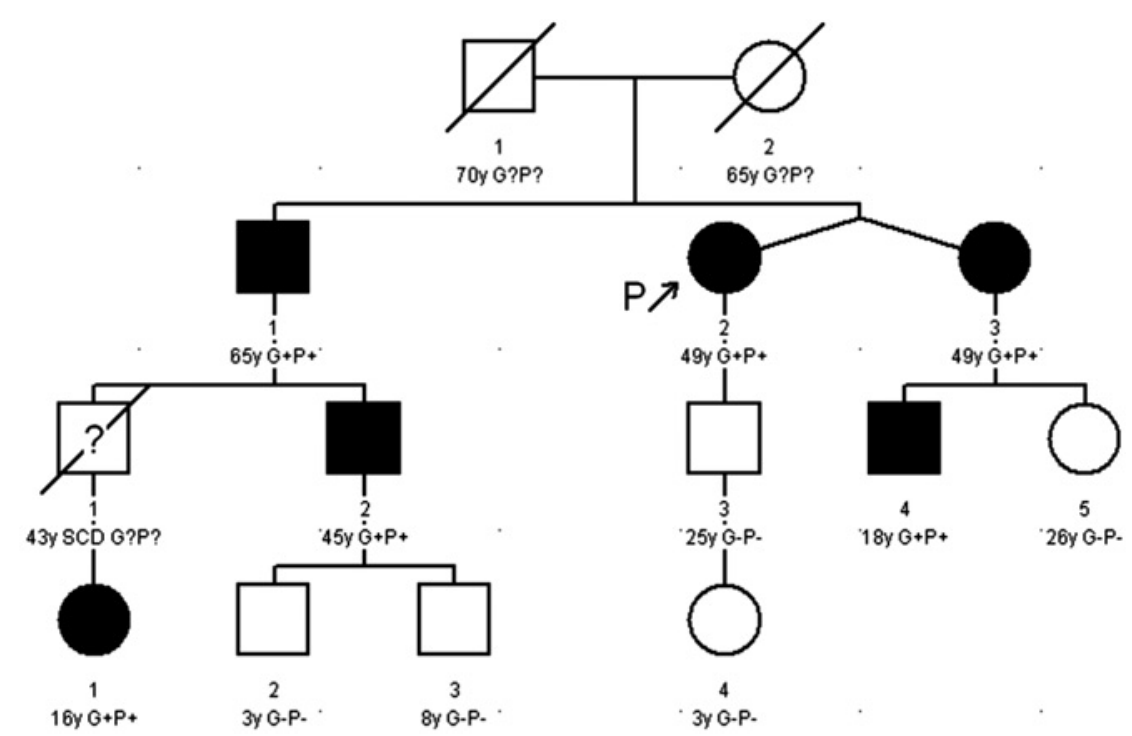

II

III

Figure 3. Pedigree of the family. Squares indicates male relatives; circles, female relatives; y, years; filled symbols, HCM patients; slants, dead members; arrow, proband; ?, the subject died before our investigation and we could not obtain clinical data and confirm his or her genotype and phenotype; SCD, sudden cardiac death; G + , positive genotype; G-, negative genotype; $\mathrm{P}+$, positive phenotype; and $\mathrm{P}-$, negative phenotype. 
SSS and a history of syncope.

\section{Discussion}

In this study, we reported the case of a monozygotic twin pair with $M Y H 7$ mutation who had variable phenotypic expressions. The findings between the twins are interesting. First, although the single site mutation of $M Y H 7$ has been previously reported in $\mathrm{HCM},{ }^{13)}$ we are the first to report the probable association of $M Y H 7$ gene mutation with phenotypic expressivity in monozygotic twins with HCM. Second, the morphologic expression of the twins was similar, but not identical. Third, the monozygotic twins presented different electrocardiographic performances. Furthermore, there were different clinical presentations and different degrees of fibrosis, as quantified by LGE and ECV, between the twins, suggesting that the twins may have different prognosis; however, this needs to be confirmed by further follow-ups.

According to review literatures, phenotypic features of HCM among monozygotic twins have been inconsistent. Maron, et al., ${ }^{15)}$ Wylie, et al., ${ }^{16)}$ and Zenovich, et al. ${ }^{17)}$ have reported that identical morphologic expression could be identified in monozygotic twins. In addition, Maron, et al. provided an explanation that genetic background is a primary factor determining the morphological expression of HCM, whereas environmental factors may have a limited impact. However, these reports have not explored the change of tissue characteristics in monozygotic twins, and other study results ${ }^{18-22)}$ have not been consistent with these reports. Palka, et al. reported there were different clinical presentations and morphological expressions in monozygotic twins with HCM. ${ }^{18)}$ Reid, et al. ${ }^{19)}$ and Ko, et al. ${ }^{20)}$ found morphological differences in the presence of LV outflow tract obstruction in monozygotic twins. Although various phenotypic features of HCM among monozygotic twins have been previously reported, quantitative assessment of fibrosis characteristics by LGE or ECV among twins has not been reported so far. In our report, although the monozygotic twins had MYH7 mutation-related HCM phenotype, the clinical presentation and fibrotic characteristics were variable.

Previous studies have suggested that methylation modification $^{23)}$ may be involved in the phenotypic expression in patients with HCM. In our report, although the monozygotic twins shared an identical mutation, their living environments and life habits were different. Therefore, the possible reason contributing to the phenotypic diversity in the monozygotic twin pair may be environmental factors and methylation modifications. Our report supports the complex pathophysiological mechanisms of HCM, especially in the course of fibrotic natural history, and suggests that epigenetics and environmental factors may affect HCM phenotype.

In conclusion, we reported different clinical presentations and different extents of myocardial fibrotic burden with similar HCM morphologic expression in a pair of monozygotic twins carrying the same MYH7 gene mutation.

\section{Disclosures}

Conflicts of interest: The authors declare no conflicts of interest.

\section{References}

1. Maron BJ, Gardin JM, Flack JM, Gidding SS, Kurosaki TT, Bild DE. Prevalence of hypertrophic cardiomyopathy in a general population of young adults echocardiographic analysis of 4111 subjects in the CARDIA study. Circulation 1995; 92: 7859.

2. Bos JM, Will ML, Gersh BJ, Kruisselbrink TM, Ommen SR, Ackerman MJ. Characterization of a phenotype-based genetic test prediction score for unrelated patients with hypertrophic cardiomyopathy. Mayo Clin Proc 2014; 89: 727-37.

3. Alfares AA, Kelly MA, Mcdermott G, et al. Results of clinical genetic testing of 2,912 probands with hypertrophic cardiomyopathy: expanded panels offer limited additional sensitivity. Genet Med 2015; 17: 880-8.

4. Hughes SE. The pathology of hypertrophic cardiomyopathy. Histopathology 2004; 44: 412-27.

5. Weng Z, Yao J, Chan RH, et al. Prognostic value of LGE-CMR in HCM: A meta-analysis. JACC Cardiovasc Imaging 2016; 9: 1392-402.

6. O'Hanlon R, Grasso A, Roughton M, et al. Prognostic significance of myocardial fibrosis in hypertrophic cardiomyopathy. J Am Coll Cardiol 2010; 56: 867-74.

7. Flett AS, Hayward MP, Ashworth MT, et al. Equilibrium contrast cardiovascular magnetic resonance for the measurement of diffuse myocardial fibrosis: preliminary validation in humans. Circulation 2010; 122: 138-44.

8. White SK, Sado DM, Fontana M, et al. T1 mapping for myocardial extracellular volume measurement by CMR: bolus only versus primed infusion technique. JACC Cardiovasc Imaging 2013; 6: 955-62.

9. Iio C, Ogimoto A, Nagai T, et al. Association between genetic variation in the scn10a gene and cardiac conduction abnormalities in patients with hypertrophic cardiomyopathy. Int Heart J 2015; 56: 421-7.

10. Feng X, He T, Wang JG, Zhao P. Asn391Thr mutation of $\beta$ myosin heavy chain in a hypertrophic cardiomyopathy family. Int Heart J 2018; 59: 596-600.

11. Wang J, Wan K, Sun J, et al. Phenotypic diversity identified by cardiac magnetic resonance in a large hypertrophic cardiomyopathy family with a single MYH7 mutation. Sci Rep 2018; 8: 973.

12. Kawai H, Morimoto SI, Takakuwa Y, et al. Hypertrophic cardiomyopathy accompanied by spinocerebellar atrophy with a novel mutation in troponin I gene. Int Heart J 2016; 57: 507-10.

13. Millat G, Chanavat V, Créhalet H, Rousson R. Development of a high resolution melting method for the detection of genetic variations in hypertrophic cardiomyopathy. Clin Chim Acta 2010; 411: 1983-91.

14. Richards S, Aziz N, Bale S, et al. Standards and guidelines for the interpretation of sequence variants: a joint consensus recommendation of the American College of Medical Genetics and Genomics and the Association for Molecular Pathology. Genet Med 2015; 17: 405-24.

15. Maron BJ, Casey SA, Almquist AK. Hypertrophic cardiomyopathy in monozygotic twins. Circulation 2002; 105: 2229.

16. Wylie L, Ramage AC, MacLeod D. Hypertrophic cardiomyopathy with shared morphology in identical twins: a case report. Scott Med J 2002; 47: 64-5.

17. Zenovich AG, Lesser JR, Hanna CA, Maron BJ. Identical twins with hypertrophic cardiomyopathy and apical aneurysm. Am J Cardiol 2006; 97: 1109.

18. Palka P, Lange A, Burstow DJ. Different presentation of hypertrophic cardiomyopathy in monozygotic twins. Heart 2003; 
89: 751 .

19. Reid JM, Houston AB, Lundmark E. Hypertrophic cardiomyopathy in identical twins. Br Heart J 1989; 62: 384-8.

20. Ko Y, Tang TK, Chen J, Hshieh Y, Wu C, Lien W. Idiopathic hypertrophic cardiomyopathy in identical twins: morphological heterogeneity of the left ventricle. Chest 1992; 102: 783-5.

21. Goh CY, Asrar Ul Haq M, Mutha V, van Gaal WJ. Synchronous cardiac arrest in monozygotic twins with hypertrophic cardio- myopathy - Is sudden cardiac death genetically preprogrammed? BMC Cardiovasc Disord 2015; 15: 16.

22. Kovács A, Molnár AÁ, Celeng C, et al. Hypertrophic cardiomyopathy in a monozygotic twin pair: similarly different. Circ Cardiovasc Imaging 2016; 9: e004794.

23. Clifford CP, Nunez DJR. Human $\beta$-myosin heavy chain mRNA prevalence is inversely related to the degree of methylation of regulatory elements. Cardiovasc Res 1998; 38: 736. 